\title{
In vivo Anticoagulant Activity of Immediate Release Tablets of Dabigatran Etexilate Mesylate Cocrystals
}

\author{
Ashwini Gawade ${ }^{1, *}$, Sanjay Boldhane ${ }^{2}$, Anil Pawar ${ }^{1}$, Rohini Pujari $^{1}$, Ashwin Kuchekar ${ }^{1}$ \\ ${ }^{1}$ School of Pharmacy, Dr. Vishwanath Karad Maharashtra Institute of Pharmacy (MIP), World Peace University, Kothrud, Pune - \\ 411038, Maharashtra, India \\ ${ }^{2}$ Development Micro Labs Limited, Micro Advanced Research Centre (Marc.), No. 58/3, Kudlu Village, Anekal Taluk, Singasandra \\ Post Bangalore - 560068, Karnataka, India
}

Received April 20, 2021; Revised August 16, 2021; Accepted August 31, 2021

\section{Cite This Paper in the following Citation Styles}

(a): [1] Ashwini Gawade, Sanjay Boldhane, Anil Pawar, Rohini Pujari, Ashwin Kuchekar, "In vivo Anticoagulant Activity of Immediate Release Tablets of Dabigatran Etexilate Mesylate Cocrystals," Advances in Pharmacology and Pharmacy, Vol. 10, No. 1, pp. 1 - 7, 2022. DOI: 10.13189/app.2022.100101.

(b): Ashwini Gawade, Sanjay Boldhane, Anil Pawar, Rohini Pujari, Ashwin Kuchekar (2022). In vivo Anticoagulant Activity of Immediate Release Tablets of Dabigatran Etexilate Mesylate Cocrystals. Advances in Pharmacology and Pharmacy, 10(1), 1 - 7. DOI: 10.13189/app.2022.100101.

Copyright $(\mathrm{O} 2022$ by authors, all rights reserved. Authors agree that this article remains permanently open access under the terms of the Creative Commons Attribution License 4.0 International License

\begin{abstract}
Dabigatran Etexilate Mesylate (DEM), a salt of prodrug dabigatran etexilate, is a potent, oral, reversible and direct thrombin inhibitor with low oral bioavailability. The present research investigation focused on the formulation of immediate release (IR) tablets of DEM cocrystals and evaluation of In vivo anticoagulant activity. The results of the study showed that the formulated IR tablets of DEM showed improved efficacy in comparison with the plain drug by enhancing the pre-compression parameters such as bulk density, tap density, Carr's index, angle of repose and Hausner's ratio and post-compression parameters like thickness and weight variation, hardness and friability, In vitro dissolution parameters. The improved efficacy was confirmed by improvement in the pharmacodynamic parameters such as cutaneous bleeding time and clotting time indicative of enhanced bioavailability of dabigatran. Thus, it can be concluded that the IR tablets of dabigatran cocrystals can be proven to be more effective in producing the anticoagulant effect in clinical practice as compared to the plain drug resulting in more patient compliance.
\end{abstract}

Keywords Dabigatran Etexilate Mesylate, Cocrystals, Anticoagulant, Immediate Release, Cutaneous Bleeding, Clotting Time

\section{Introduction}

Anticoagulant agents are used worldwide to regulate blood coagulation in both healthy and diseased conditions such as cancer, diabetes mellitus and cardiovascular diseases [1]. Despite the development, proven efficacy, and advancement of these drugs, most of them are associated with several undesirable adverse effects such as bleeding of mild or severe in vital organs, while some are associated with drug and food interactions [2]. Warfarin-based therapy requires constant drug plasma concentration monitoring and its indirect action mechanism affects various coagulation factors showing a high risk of bleeding [3].

Heparin and its fractional forms are not useful in the treatment of chronic thrombotic diseases due to its poor oral bioavailability and high molar mass [4]. Modern non-vitamin K antagonist oral anticoagulants achieve their effect through direct inhibition of key coagulation factors, such as thrombin or FXa [5]. They do not possess the aforementioned issues. Despite this, their medical use is associated with the risk of life-threatening bleeding events, which require urgent administration of a specific reversal agent [6]. Hence, research interest in the discovery of novel anticoagulant drugs from other chemical classes with less toxicity and fewer side effects has been increasing. 
Dabigatran etexilate is a low-molecular-weight non-peptide prodrug that gets converted to its active form, dabigatran by ubiquitous esterases upon oral administration. It is a univalent, competitive, reversible and potent direct inhibitor of thrombin, the final effector in blood coagulation [7]. Thrombin molecule consists of one active site and two secondary binding exosites. Exosite 1 acts as a domain for binding of substrates such as fibrin to enhance orientation for binding to the active site, whereas, exosite 2 acts as a domain for binding of heparin. Dabigatran acts by binding to the active site of thrombin, thus inactivating both fibrin-bound and unbound thrombin [8,9]. The ability to inhibit fibrin-bound thrombin is an important theoretical advantage of dabigatran over the heparins because bound thrombin can continue to trigger thrombus expansion $[10,11]$. Other advantages of dabigatran in comparison with other anticoagulants include predictable anticoagulant effect, rapid onset of action, low potential for drug-drug and drug-food interactions and target-specificity towards coagulation enzyme. By inhibiting thrombin, dabigatran prevents the conversion of fibrinogen into fibrin, positive feedback amplification of coagulation activation, cross-linking of fibrin monomers, platelet activation and inhibition of fibrinolysis [12,13].

Dabigatran Etexilate Mesylate (DEM) is a salt form of the prodrug dabigatran etexilate. It belongs to BCS Class II drugs i.e., low solubility and high permeability class with absolute bioavailability of about 3-7 \% followed by oral administration. It has an elimination half-life of 12-17 hours, with a dose of $75 \mathrm{mg}, 110 \mathrm{mg}$, and $150 \mathrm{mg}$. It is prone to acid and in the presence of moisture, it undergoes degradation by hydrolytic pathways. DEM has strong $\mathrm{pH}$-dependent solubility, in acid media, decreases with an increase in $\mathrm{pH}$ and is practically insoluble in basic media $[14,15]$. Hence, it is necessary to enhance the aqueous solubility and dissolution rate of DEM. It is also essential to maintain the stability of DEM in basic $\mathrm{pH}$ to achieve faster onset of action, minimize the variability in absorption and improve its overall oral bioavailability. A technique called "cocrystallization" has recently gained significant attention in drug delivery by improving the physicochemical properties of drugs such as melting point, solubility, dissolution rate, stability, and bioavailability without changing its chemical structure [16-18].

The attempt is made to formulate the immediate release tablet dosage form of DEM cocrystals to improve the rate of dissolution, absorption, bioavailability and efficacy of the DEM and to carry out its In vivo anticoagulant activity to confirm the same.

\section{Materials and Methods}

\subsection{Material}

Dabigatran Etexilate Mesylate was received as a gift sample from Microlab research lab Bangalore India. Dabigatran etixilate mesylate cocrystals are prepared in-house. Microcrystalline cellulose was received as a gift sample from Colorcorn Asia Pvt. Ltd., Goa, India. Crosspovidone was received as a gift sample from Concept Pharmaceutical Pvt. Ltd., Aurangabad, India. All other chemicals and reagents are of analytical grades.

\subsection{Animals}

For acute oral toxicity studies female Swiss albino mice (22-25 g) were used and for In vivo anticoagulant activity, Wistar rats (150-200 g) were used for In vivo anticoagulant activity. They were procured from the National Toxicology Centre, Pune. Animals were housed in polypropylene cages $(38 \mathrm{~cm} \times 23 \mathrm{~cm} \times 10 \mathrm{~cm})$ under standard laboratory conditions at $25 \pm 2{ }^{\circ} \mathrm{C}$ temperature, $60 \pm 5 \% \mathrm{RH}$ and $24{ }^{\circ} \mathrm{C} ; 12: 12 \mathrm{~h}$ dark/light cycle with free access to standard pelleted diet (Pranav Agro Industries Ltd., Sangli, India) and water ad libitum. Animals were fasted overnight on the day of study. Proper care and maintenance of the animals was undertaken following the guidelines of the Committee for Prevention, Control and Supervision of Experimental Animals, Govt. of India.

\subsection{Ethical Clearance}

All the studies involving animal experiments were carried out in accordance with the experimental protocols approved by the Institutional Animal Ethics Committee of School of Pharmacy, Dr. Vishwanath Karad, MIT WPU, M.S. India (Protocol No. MIP/IAEC/2019-20/M2/01).

\subsection{Preparation of DEM Cocrystals}

DEM co-crystals synthesis was performed using the solvent evaporation technique. Screening of formation of DEM co-crystals was performed by various coformers in an optimal molar ratio $(1: 1,1: 2$ and 1:3). A mixture of $1: 1$ DEM and Tartaric acid was dissolved in ethanol and continually stirred at $40-50{ }^{\circ} \mathrm{C}$ for $15 \mathrm{~min}$. The solvent evaporated at $60-65{ }^{\circ} \mathrm{C}$ when stored for $3 \mathrm{~h}$ in a hot air oven. Cocrystals were triturated in mortar and pestle and stored at room temperature [18-21]. 


\subsection{Formulation of IR Tablets of DEM Cocrystals}

IR tablets of DEM cocrystals were prepared by direct compression method using crospovidone (CP) as super-disintegrant to improve the dissolution of the drug. HPMC K4M was used as a binder. Microcrystalline cellulose (MCC) and lactose monohydrate were used as diluents, respectively. And Talc was used as a glidant. DEM cocrystals equivalent to $75 \mathrm{mg}$ of DEM and all the excipients except magnesium stearate were taken in mortar. All the ingredients were co-ground in a pestle and motor and then aerosil and magnesium stearate were added and mixed for 10 minutes. Then powder blend was mixed well for 15 to $30 \mathrm{~min}$. The blends were passed through the \# 80 sieve. Lubrication was done using magnesium stearate. The final blend was compressed on a Remake Mini Press II D Tooling 8 station compression machine equipped with concave punches to a weight of $300 \mathrm{mg} /$ tablet. The compressed tablets were evaluated for pre- and post-compression parameters [22].

\subsection{Physical Evaluation of IR Tablets of DEM Cocrystals}

The prepared blends were evaluated for pre-compression parameters like bulk density, tap density, Carr's index, angle of repose, and Hausner's ratio and post-compression parameters like thickness and weight variation, hardness and friability, In vitro dissolution study [23].

\subsection{Stability Studies of Tablets}

Studies were carried out for 45 days for the optimized batches of DEM cocrystals IR tablets at a temperature $40 \pm 2{ }^{\circ} \mathrm{C} / \mathrm{RH} 75 \pm 5 \%$ [24]

\subsection{Acute Oral Toxicity Study of Tablets}

The acute oral toxicity test was performed using the Acute Toxicity Class (ATC) method according to the Organization of Economic Co-operation and Development (OECD) guideline 423. Female Swiss albino mice were weighed and randomly divided into two groups (6 mice (group). The first group served as the test group and was administered orally with the suspension of powdered DEM cocrystals IR tablets in distilled water at a single dose of $2000 \mathrm{mg} / \mathrm{kg}$ body weight. The second group served the control group and received only distilled water at a volume of $10 \mathrm{ml} / \mathrm{kg}$ body weight. Observations were noted at $1,2,4$ and $6 \mathrm{~h}$ after administration of test substance and recorded systematically. The visual observations like changes in the skin and fur, eyes and mucous membranes were recorded. Further, respiratory, circulatory, autonomic and central nervous systems, as well as somatomotor activity and behavioral pattern, were observed. The number of survivors was recorded initially after $48 \mathrm{~h}$ and then a further 14 days with once-a-day observation [25].

\subsection{Evaluation of In vivo Anticoagulant Activity of IR Tablets of Dabigatran Cocrystals Using Cutaneous Bleeding Time Model}

Wistar rats were divided into three groups $(\mathrm{n}=6)$. Group I was considered as control group and was administered with distilled water at a dose of $1 \mathrm{ml} / \mathrm{kg}$. Group II was administered with the suspension of plain DEM in distilled water at a dose of $50 \mathrm{mg} / \mathrm{kg}$ orally while group III was administered with the suspension of powdered tablets from an optimized batch of IR tablets of DEM cocrystals at the dose of $50 \mathrm{mg} / \mathrm{kg}$ orally. After one hour, the rats of all groups were anesthetized by intraperitoneal administration of ketamine $(100 \mathrm{mg} / \mathrm{kg})$ and xylazine $(10$ $\mathrm{mg} / \mathrm{kg}$ ) and placed individually in a plastic rat holder with several openings from one of which the animal tail was taken out. The tail was cleaned properly with water-wetted cotton. Then incision $(10 \mathrm{~mm}$ long and 1.5 $\mathrm{mm}$ deep) was made with a scalpel between 8 and $9 \mathrm{~cm}$ from the tip of the tail (Figure 1). The bleeding time was assessed at intervals of $15 \mathrm{~s}$ [26].

\subsection{Evaluation of In vivo Anticoagulant Activity of IR Tablets of Dabigatran Cocrystals Using Clotting Time Model}

Wistar rats were divided into three groups $(\mathrm{n}=6)$. Group I was considered as control group and was administered with distilled water at a dose of $1 \mathrm{ml} / \mathrm{kg}$. Group II was administered with the suspension of plain DEM in distilled water at a dose of $50 \mathrm{mg} / \mathrm{kg}$ orally while group III was administered with the suspension of powdered tablets from the optimized batch of IR tablets of DEM cocrystals at the dose of $50 \mathrm{mg} / \mathrm{kg}$ orally. After one hour, the rats of all groups were anesthetized by intraperitoneal administration of ketamine $(100 \mathrm{mg} / \mathrm{kg})$ and xylazine $(10$ $\mathrm{mg} / \mathrm{kg}$ ) and blood was withdrawn into the capillary tube through retro-orbital route to fill $3 / 4^{\text {th }}$ of the capillary tube. Clot formation was checked after every 30 seconds by breaking a piece of a capillary tube and slightly stretching apart the two ends of the broken capillary tube. The time at which a thread-like structure called fibrin extends between the two ends of the capillary tube is noted down as clotting time [26].

\subsection{Statistical ANALYSIS}

The results were expressed as Mean \pm SEM $(n=6)$. Comparison between the groups was made by one-way analysis of variance (ANOVA) followed by Tukey's Kramer Multiple Comparison test using Instat Graph Pad software (version-3). 


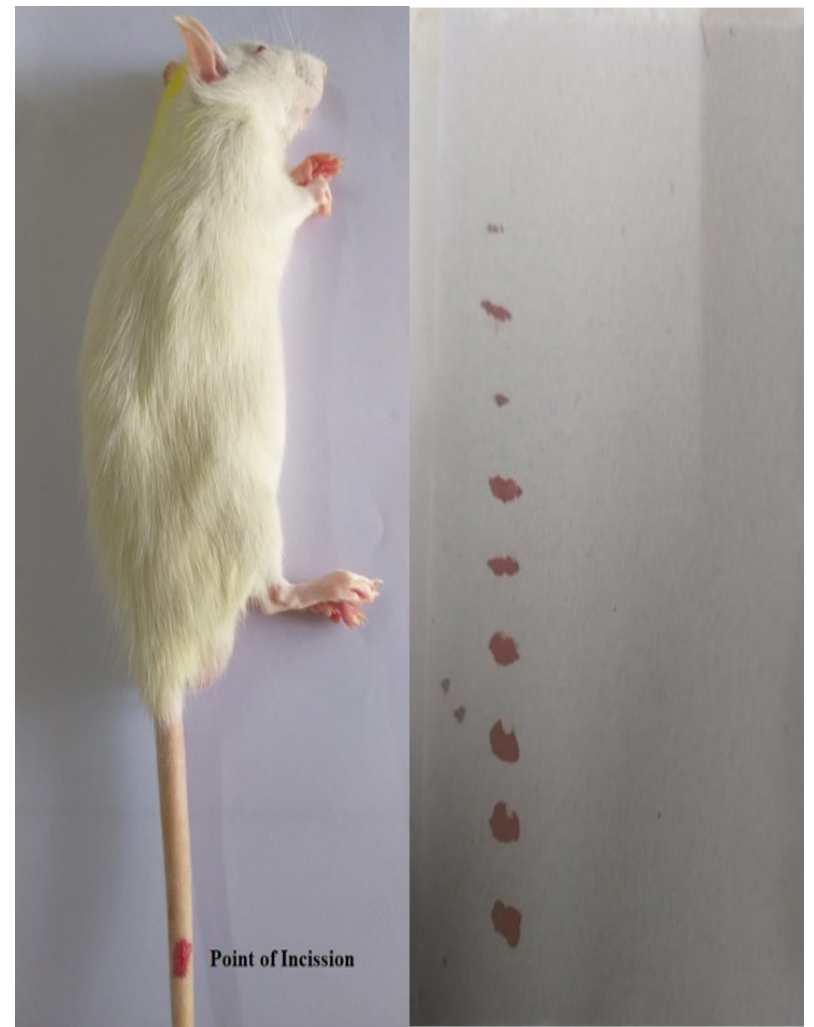

Figure 1. Evaluation of In vivo anticoagulant activity of IR tablets of dabigatran cocrystals using cutaneous bleeding time model

\section{Results and Discussion}

\subsection{Evaluation of Pre-Compression Parameters of IR Tablets of DEM Cocrystals}

Bulk density was found in the range of $0.291 \pm 0.012$ to $0.363 \pm 0.014 \mathrm{~g} / \mathrm{cm}^{3}$, tapped density between $0.346 \pm 0.014$ and $0.514 \pm 0.023 \mathrm{~g} / \mathrm{cm}^{3}$, using the above two density data, Hausner's Ratio and Compressibility Index (CI) were calculated. The powder blends of all formulations with Hausner's ratio $<1.25$ indicated better flow properties. The compressibility index was found to range between 0.01 and $0.22 \%$ and the compressibility and flowability data indicated an excellent flowability of all powder blends. The better flowability of all powder blends was also evidenced from the angle of repose (in the range of 25.66 \pm 2.16 to $30.45 \pm 1.49$ ) which is below $40 \theta^{\circ}$, indicating good flowability.

\subsection{Evaluation of Post-Compression Parameters of IR Tablets of DEM Cocrystals}

Tablet weights varied between $282 \pm 0.06$ and 310 $\pm 0.06 \mathrm{mg}$, hardness between 5 and $7 \mathrm{~kg} / \mathrm{cm}^{2}$ (average 6 $\mathrm{kg} / \mathrm{cm}^{2}$ ), thickness between 3.20 and $3.60 \mathrm{~mm}$ (average $3.4 \mathrm{~mm}$ ) and friability ranged from $0.04 \%$ and $0.13 \%$ (average $0.40 \%$ ). The results of drug content and physical examination of all formulations were found to be within the official limits. Among all the 13 batches of formulations Batch F8 showed all the results within specification and was considered to be the optimized batch.

\subsection{In-vitro Dissolution Study of IR Tablets of DEM Cocrystals}

The data showed that the optimized batch shows a good dissolution profile and the same was selected for further study for in vivo activity. The optimized batch showed the highest drug release $100.5 \%$ at 60 minutes when compared to all other batches. The obtained results of In vitro drug release showed a relationship between the binder and disintegrant concentration and the In vitro release of DEM cocrystals IR tablets.

\subsection{Stability Study of IR tablets of DEM Cocrystals}

All physical parameters of the optimized batch of tablets were found to be within the standard range when kept under accelerated stability conditions during the stability studies indicating that the formulation showed good stability.

\subsection{Acute Oral Toxicity Test of IR Tablets of DEM Cocrystals}

The results showed that immediate release tablets of DEM cocrystals were found to be safe without any mortality and morbidity up to the dose of $300 \mathrm{mg} / \mathrm{kg}$.

\subsection{Effect of IR Tablets of DEM Cocrystals on In vivo Anticoagulant Activity Using Cutaneous Bleeding Model}

Bleeding time is the time elapsed from the moment the tail is incised to the first arrest of bleeding. Compared with the control group, all treatments significantly $(\mathrm{P}<0.001)$ prolonged the cutaneous bleeding time. Untreated animals (control group) showed a mean bleeding time of 47 secs while the animals treated with test drug suspensions showed comparatively increased mean bleeding time. However, as compared to plain DEM (mean bleeding time -105 secs), the DEM Tablets (mean bleeding time- 157 secs) exhibited more significant $(\mathrm{P}<0.001)$ efficacy in enhancing the bleeding time which correlated well with pharmacokinetic data (Figure 2). Hence, we could conclude that the developed formulation exhibited better anticoagulation activity than plain drug suspension by improving the oral bioavailability of DEM $[8,18]$.

\subsection{Effect of IR Tablets of DEM Cocrystals on In vivo Anticoagulant Activity Using Clotting Time Model}

Compared with the control group, all treatments 
significantly $(\mathrm{P}<0.001)$ prolonged the clotting time. Untreated animals (control group) showed a mean clotting time of 1.49 mins while the animals treated with test drug suspensions showed comparatively increased mean clotting time. However, as compared to plain DEM (mean clotting time -2.53 mins), the DEM Tablets (mean clotting time -3.08 mins) exhibited more significant
$(\mathrm{P}<0.001)$ efficacy in enhancing the bleeding time which correlated well with pharmacokinetic data (Figure 3). Hence, we could conclude that the developed formulation exhibited better anticoagulation activity than plain drug suspension by improving the oral bioavailability of DEM $[27,28]$.

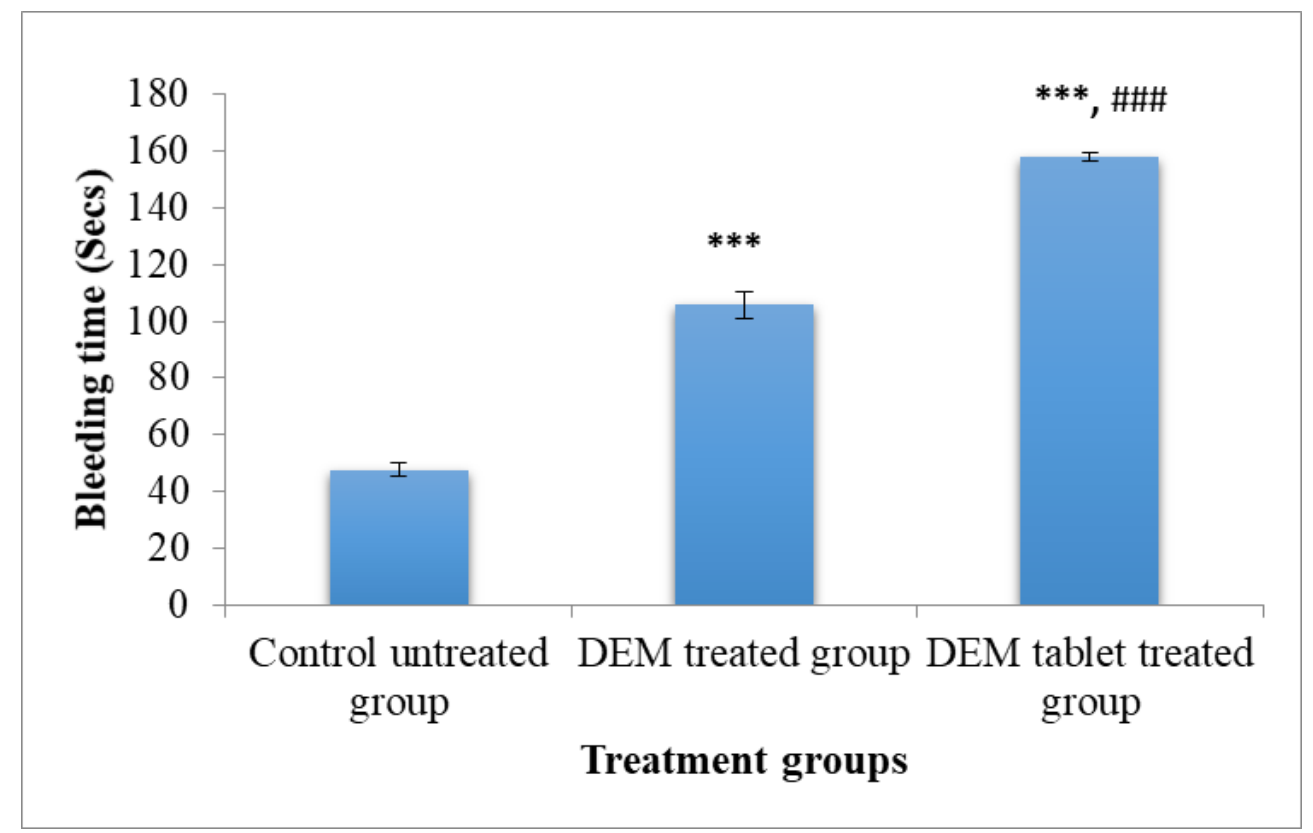

Results were expressed as mean \pm SEM. Comparison between the groups was made by one-way analysis of variance (ANOVA) followed by Tukey's test *, \#-P $<0.05,{ }^{* *}, \# \#-\mathrm{P}<0.01,{ }^{* * *}$, \#\#\#-P<0.001; *- DEM and DEM tablets treated groups against control untreated group; \#- DEM tablet treated group against $\mathrm{DEM}$ treated group.

Figure 2. Comparative bleeding time in control untreated, DEM treated and DEM cocrystals IR tablet treated group

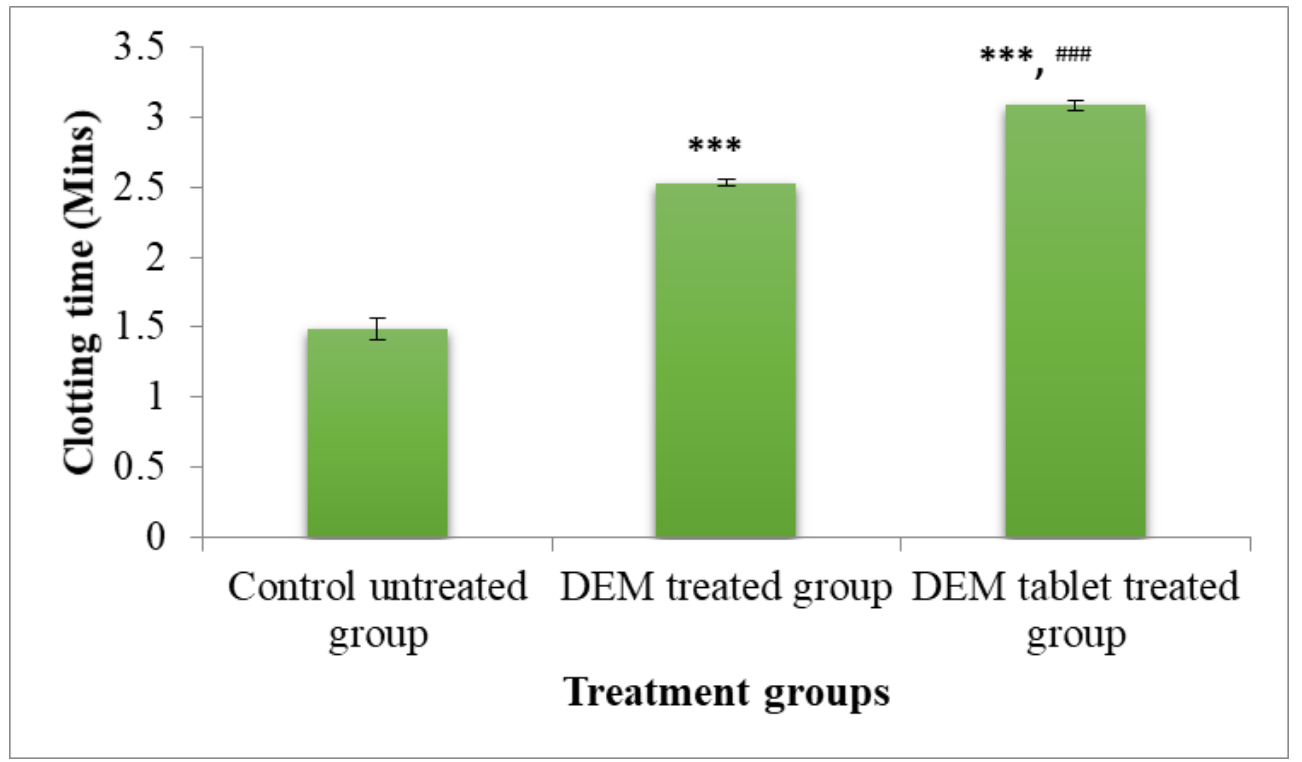

Results were expressed as mean \pm SEM. Comparison between the groups was made by one-way analysis of variance (ANOVA) followed by Tukey's test *, \#-P $<0.05,{ }^{* *}, \# \#-\mathrm{P}<0.01,{ }^{* * *}, \# \# \#-\mathrm{P}<0.001 ;{ }^{*}$ - DEM and DEM tablets treated groups against control untreated group; \#- DEM tablet treated group against DEM treated group.

Figure 3. Comparative bleeding time in control untreated, DEM treated and DEM cocrystals IR tablet treated group 


\section{Conclusion}

It can be concluded from the present study that the $\mathrm{pH}$-dependent solubility and short absorption window of DEM was overcome by formulating it in cocrystal form and converting it into IR tablet formulation. The formulation showed significant improvement in the rate of dissolution, absorption and bioavailability of DEM, which in turn improved its In vivo anticoagulant activity by virtue of enhanced cutaneous bleeding time and clotting time. Thus, the improvement in pharmacokinetic and pharmacodynamic parameters of DEM would enhance the efficacy and increase patient compliance.

\section{Acknowledgements}

This study was supported by the School of Pharmacy, Dr. Vishwanath Karad, MIT, WPU, Kothrud, Pune-411038, Maharashtra, India by providing the required experimental facilities.

\section{REFERENCES}

[1] Wadhera R. K., Russell C. E., G. Piazza, "Warfarin versus novel oral anticoagulants how to choose?," Circulation, vol. 130, pp. e191-193, 2014. https://doi.org/10.1161/CIRCULATIONAHA.114.010426

[2] Compton K., "Blood thinners: anticoagulants \& antiplatelet drugs," Drugwatch, South Orange Avenue, Orlando, FL. 2019. doi: 10.2147/JEP.S218261

[3] Bryan J., "Short acting and reversible effects made heparin a great anticoagulant," Pharm J, vol. 290, pp. 188, 2013. doi: 10.1211/PJ.2013.11116881

[4] Truong J. T., S. L. Booth, "Emerging issues in vitamin K research," J Evid Based Complement Altern Med, vol. 16, no. 1, pp. 73-79, 2011. doi: 10.1177/1533210110392953

[5] Blann A. D., C. W. Khoo, "The prevention and treatment of venous thromboembolism with LMWHs and new anticoagulants," Vasc Health Risk Manag, vol. 5, pp. 693-704, 2009. doi: 10.2147/VHRM.S4621

[6] Huntington J. A., T. P. Baglin, "Targeting thrombin: rational drug design from natural mechanism," Trends Pharmacol Sci, vol. 24, pp. 589-595, 2003. doi: 10.1016/j.tips.2003.09.002

[7] Eisert W. G., Hauel N., Stangier J., Wienen W., Clemens A., J. van Ryn, "Dabigatran: an oral novel potent reversible nonpeptide inhibitor of thrombin," Arterioscler Thromb Vasc Biol, vol. 30, pp. 1885-1889. doi: 10.1161/ATVBAHA.110.203604

[8] van Ryn J., Hauel N., Waldman L., W. Wienen, "Dabigatran inhibits both clot-bound and fluid-phase thrombin in vitro: comparison to heparin and hirudin [abstract 570]," Arterioscler Thromb Vasc Biol, vol. 28, pp. e136-e137, 2008. https://doi.org/10.1182/blood.V110.11.3 998.3998

[9] Weitz J. I., Hudoba M., Massel D., Maraganore J., J. Hirsh, "Clot-bound thrombin is protected from inhibition by heparin-antithrombin III but is susceptible to inactivation by antithrombin III-independent inhibitors," J Clin Invest, vol. 86, pp. 385-391, 1990. https://doi.org/10.1172/JCI114 723

[10] Weitz J. I., Leslie B., M. Hudoba, "Thrombin binds to soluble fibrin degradation products where it is protected from inhibition by heparin-antithrombin but susceptible to inactivation by antithrombin-independent inhibitors," Circulation, vol. 97, pp. 544-552, 1998. doi: 10.1161/01.cir.97.6.544

[11] Stangier J., Rathgen K., Stahle H., Gannser D., W. Roth, "The pharmacokinetics, pharmacodynamics, and tolerability of dabigatran etexilate, a new oral direct thrombin inhibitor, in healthy male subjects," Br J Clin Pharmacol, vol. 64, pp. 292-303, 2007. doi: 10.1111/j.1365-2125.2007.02899.x

[12] Blech S., Ebner T., Lidwig-Schwellinger E., Sangier J., W. Roth. "The metabolism and disposition of the oral direct thrombin inhibitor, dabigatran, in humans," Drug Metab Disposition, vol. 36, pp. 386-399, 2008. doi: 10.1124/dmd.107.019083

[13] Burness C. B., K. McKeage, "Dabigatran etexilate: a review of its use for the prevention of venous thromboembolism after total hip or knee replacement surgery," Drugs, vol. 72, no. 7, pp. 963-986, 2012. https://doi.org/10.2165/1120908 0-000000000-00000

[14] Baumann K. L. M., Morton C. T., D. J. Dries, "New anticoagulants: A concise review," In Journal of Trauma and Acute Care Surgery, vol. 73, no. 4, pp. 983-992, 2012. https://doi.org/10.1097/TA.0b013e318265cf9e

[15] Patole T., A. Deshpande, "Co-Crystallization-A technique for solubility enhancement," International journal of pharmaceutical sciences and research, vol. 5, no. 9, pp. 3566-3576, 2014. https://doi.org/10.13040/IJPSR.0975-82 32.5(9).3566-76

[16] Perlovich G. L., A. N. Manin, "Design of pharmaceutical cocrystals for drug solubility improvement," Russian Journal of General Chemistry, vol. 84, pp. 407-414, 2014. https://doi.org/10.1134/S107036321402042X

[17] Pol S., Nawale R., Puranik P., Chalak H., H. Pol, "Scientific coformer screening, preparation and evaluation of Dabigatran Etexilate Mesylate cocrystal"' Asian Journal of Pharmacy and Pharmacology, vol. 4, no. 6, pp. 810-820, 2018. https://doi.org/10.31024/ajpp.2018.4.6.15

[18] Gawade A. R., and S. Boldhane, "Solid dosage form development of dabigatran etexilate mesylate with increased solubility and dissolution using co-crystallization," Int J Pharm Sci \& Res, vol. 11, no. 5, pp. 2899-2905, 2020. doi: 10.13040/IJPSR.0975-8232.11(5).2899-05

[19] Karimi-Jafari M., Luis P., Gavin M. W., M. C. Denise, Creating cocrystals: A review of pharmaceutical cocrystal preparation routes and applications. Crystal Growth and Design, vol. 18, no. 10, pp. 6370-6387, 2018. https://doi.org/10.1021/acs.cgd.8b00933 
[20] Duggirala N. K., Miranda L. P., Örn A., J. Z. Michael, "Pharmaceutical Cocrystals: Along the path to improved medicines," Chemical Communications, vol. 52, pp. 640-655, 2016. https://doi.org/10.1039/C5CC08216A

[21] Newman A., Susan M. R., Z. George. "Coamorphous active pharmaceutical ingredient-Small molecule mixtures: Considerations in the choice of coformers for enhancing dissolution and oral bioavailability," Journal of Pharmaceutical Sciences, vol. 107, no. 1, pp. 5-17, 2018. doi: 10.1016/j.xphs.2017.09.024

[22] Pawar A. P., Munde P. L., Bothiraja C., A. B. Kuchekar, "Development of ranolazine loaded floating biomaterial gellan beads using box-behnken factorial design," Materials Technology, vol. 30, no. 1, pp. 33-42, 2015. https://doi.org/10.1179/1753555714Y.0000000199

[23] Kuchekar A. B., A. P. Pawar, "Screening of factors using plackett burman design in the preparation of capecitabine-loaded nano polymeric micelles," International Journal of Pharmacy and Pharmaceutical Sciences, vol. 6, no. 5, pp. 489-496, 2014. https://innovareacademics.in/journal/ijpps/Vol6Issue5/942 2.pdf

[24] Lipnick R. L. et al. Comparison of the Up-and-down, Conventional LD50, and Fixed-Dose Acute Toxicity
Procedures. Food and Chemical Toxicology, vol. 33, no. 3, pp. 223-231, 1995.doi: 10.1016/0278-6915(94)00136-c

[25] Brown D. G., Eric C. W., W. L. Elliot. "A Review of Traditional and Novel Oral Anticoagulant and Antiplatelet Therapy for Dermatologists and Dermatologic Surgeons.," Journal of the American Academy of Dermatology, vol. 72, no. 3, pp. 524-534, 2015. doi: 10.1016/j.jaad.2014.10.027

[26] Reilly P. A. et al. "The Effect of Dabigatran Plasma Concentrations and Patient Characteristics on the Frequency of Ischemic Stroke and Major Bleeding in Atrial Fibrillation Patients: The RE-LY Trial (Randomized Evaluation of Long-Term Anticoagulation Therapy)," Journal of the American College of Cardiology, vol. 63, no. 4, pp. 321-328, 2014. doi: 10.1016/j.jacc.2013.07.104

[27] Lee, W., Yoo, H., Ku, S. K., Kim, J. A., \& Bae, J. S. Anticoagulant activities of piperlonguminine in vitro and in vivo. BMBvreports, 46(10), 484-489, 2013. https://doi.org/10.5483/bmbrep.2013.46.10.028.

[28] Gogoi, D., Arora, N., Kalita, B. et al. Anticoagulant mechanism, pharmacological activity, and assessment of preclinical safety of a novel fibrin(ogen)olytic serine protease from leaves of Leucas indica. Sci Rep 8, 6210, 2018. https://doi.org/10.1038/s41598-018-24422-y 